\title{
Vancomycin-associated linear IgA disease mimicking toxic epidermal necrolysis*
}

\author{
Amanda Regio Pereira ${ }^{1}$ \\ Jhonatan Rafael Siqueira Pinheiro ${ }^{1}$ \\ Milvia Maria Simões e Silva Enokihara ${ }^{1}$
}

\author{
Luis Henrique Barbizan de Moura ${ }^{1}$ \\ Victor Pavan Pasin ${ }^{1}$ \\ Adriana Maria Porro ${ }^{1}$
}

DOI: http:/ /dx.doi.org/10.1590/abd1806-4841.20164665

\begin{abstract}
Linear IgA dermatosis is a rare subepidermal autoimmune blistering disease characterized by linear deposition of IgA along the basement membrane zone. In the last three decades, many different drugs have been associated with the druginduced form of the disease, especially vancomycin. We report a case of vancomycin-induced linear IgA disease mimicking toxic epidermal necrolysis. The aim of this work is to emphasize the need to include this differential diagnosis in cases of epidermal detachment and to review the literature on the subject and this specific clinical presentation.
\end{abstract}

Keywords: Linear IgA bullous dermatosis; Drug eruptions; Vancomycin

\section{INTRODUCTION}

Linear IgA dermatosis (LAD) is a rare autoimmune mucocutaneous blistering disease characterized immunohistopathologically by subepidermal blister and linear deposition of IgA along the basement membrane zone (BMZ) on direct immunofluorescence (DIF). It is classified as spontaneous and drug-induced (DI-LAD) forms. ${ }^{1}$ Since the first publication about DI-LAD in 1981, more than one hundred cases have been reported associating different drugs with the disease, especially vancomycin. ${ }^{2,3}$

Annular or polycyclic plaques and papules with blistering around the edges (string of pearls sign) is the classic presentation of LAD, occurring usually in childhood. ${ }^{4}$ Development of LAD in adulthood can be clinically polymorphic, mimicking dermatitis herpetiformis (DH), bullous pemphigoid (BP), pemphigus vulgaris, erythema multiforme and toxic epidermal necrolysis (TEN). ${ }^{5}$

DI-LAD tends to be more severe, extensive and atypical than spontaneous LAD. In a recent study with an expressive number of cases for a rare condition, the frequencies of mucosal involvement, presence of target or target-like lesions and the string of pearls sign did not differ between the two forms of the disease. However, Nikolsky's sign and large erosions were significantly more frequent in patients with DI-LAD. ${ }^{1}$

Few cases have been published of the rare and dramatic TEN-like clinical presentation of the disease. ${ }^{5}$ This report is justified by its iconographic exuberance, rarity and the need to include the disease in the differential diagnosis of patients with epidermal detachment.

\section{CASE REPORT}

A 75-year-old male patient was hospitalized for surgical myocardial revascularization. Past medical history included high blood pressure, diabetes, dyslipidemia and ischemic stroke. In the previous three days, he had developed painful oral ulcers, tense and flaccid bullae and vesicles over the limbs and trunk. We also identified erosions on the buttocks and a positive Nikolsky's sign (Figures 1 and 2). Abrupt onset and rapid progression of the lesions were observed. The patient had been under empiric therapy with intravenous vancomycin and ceftriaxone since the day of the surgery, ten days before the lesions started to appear. The remaining drugs in use were enoxaparin, tramadol, amiodarone and medicines under continuous and chronic prescription (losartan, aspirin, propranolol, amlodipine, clonidine and simvastatin). The vancomycin level was $31.7 \mu \mathrm{g} / \mathrm{ml}$, which is above the recommended therapeutic concentration $(15-20 \mu \mathrm{g} / \mathrm{ml})$.

Considering the clinical picture and drug history, vancomycin-induced LAD was suspected. TEN and BP were considered in the differential diagnosis. Biopsy of an intact vesicle revealed subepidermal cleavage with neutrophilic infiltrate (Figure 3). DIF on perilesional skin demonstrated linear IgA deposition at the BMZ and negative IgG, IgM and C3 fluorescence (Figure 4).

New lesions stopped appearing eight days after antibiotic discontinuation and administration of prednisone $0.5 \mathrm{mg} / \mathrm{Kg} / \mathrm{d}$. Complete healing was observed after twelve days. However, the patient died of sepsis and respiratory failure two weeks after total reepithelization of the skin.

Received on 25.04.2015

Approved by the Advisory Board and accepted for publication on 27.05.2015

Work performed at the Universidade Federal de São Paulo (UNIFESP) - São Paulo-SP - Brazil.

Financial Support: None.

Conflict of Interest: None.

1 Universidade Federal de São Paulo (Unifesp) - São Paulo (SP), Brazil.

(C)2016 by Anais Brasileiros de Dermatologia 

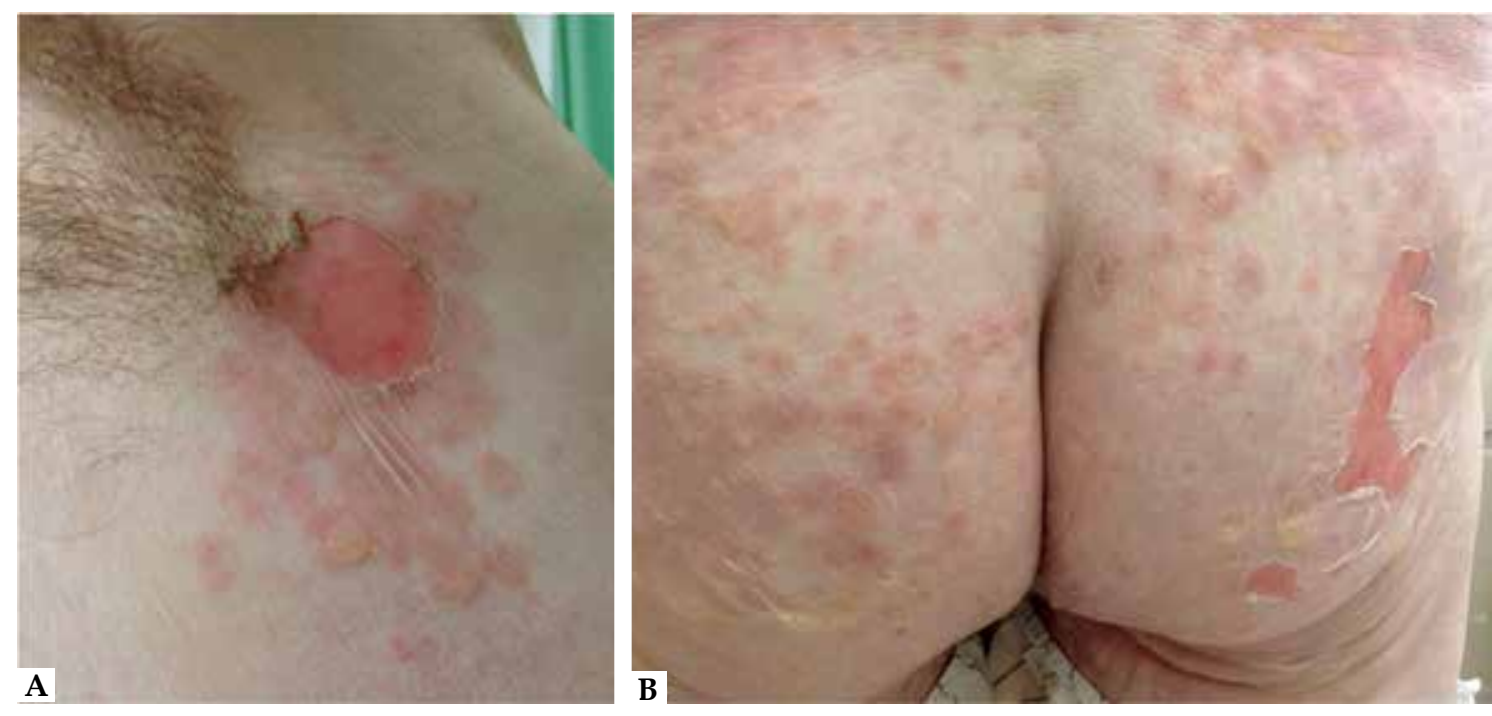

Figure 1:

A) Axillary region with flaccid bullae and erosion. B) Confluent flaccid blisters and vesicles with erosions and epidermal detachment on the buttocks

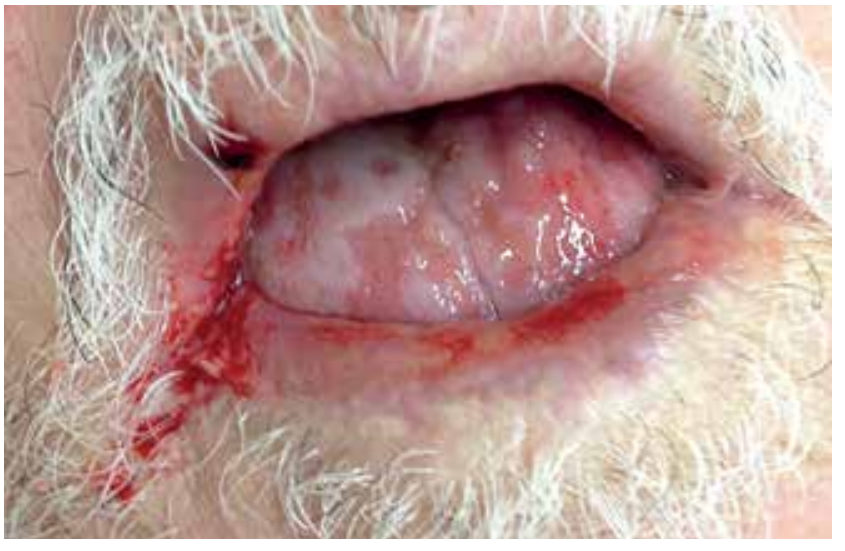

FIGURE 2: Extensive involvement of the oral mucosa and lips with erosions and friability

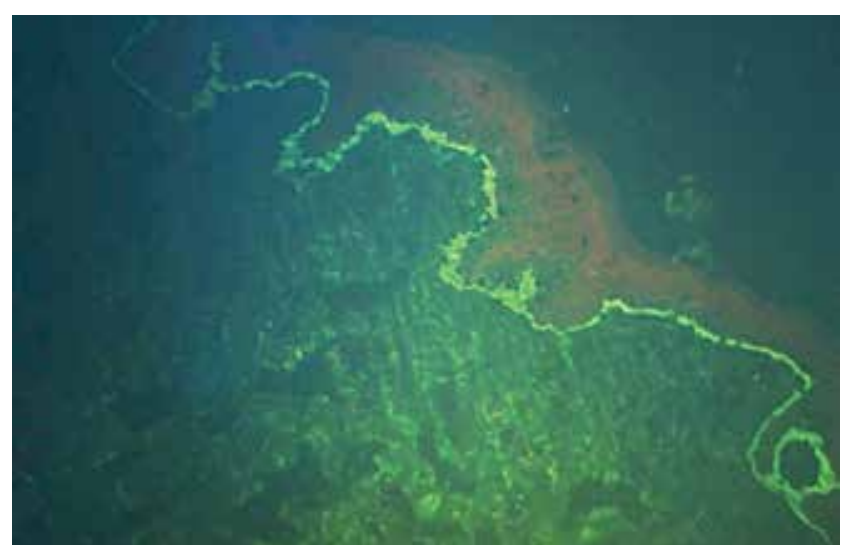

FIGURE 4: Direct immunofluorescence on perilesional skin revealing linear IgA deposition at the basement membrane zone
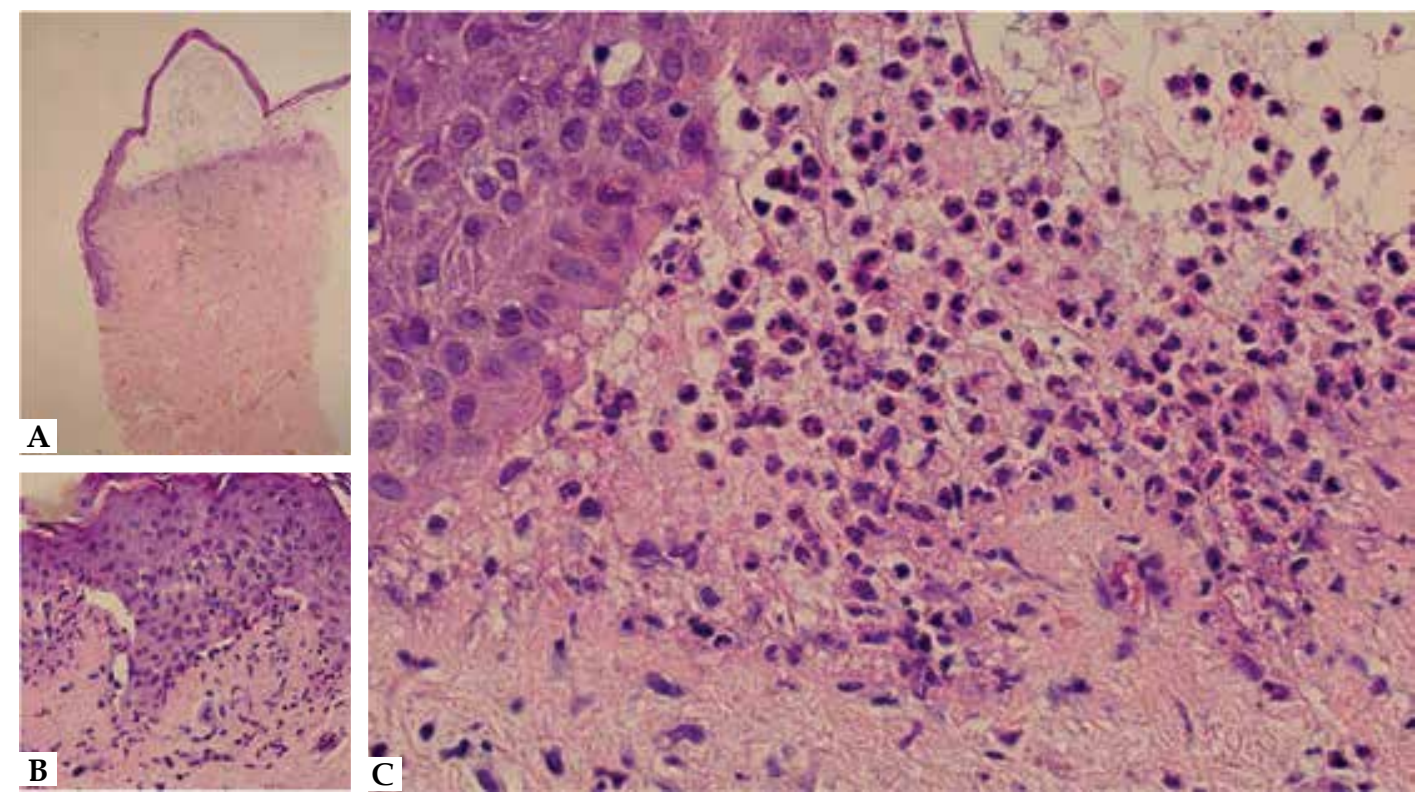

Figure 3:

Histopathology of an intact vesicle (hematoxylin and eosin). A and B) Subepidermal cleavage - 40x and 400x magnification; C) Neutrophilic infiltrate - 400x magnification 


\section{DISCUSSION}

Up to 2013, 16 cases of DI-LAD mimicking TEN had been reported. These cases were reviewed by Kakar et al., who found an average age of 69 years, variable mucous involvement (8/13), positive Nikolsky's sign in 8 out of 10 cases and affected body surface area ranging from $15 \%-90 \%$. All surviving patients showed resolution of lesions two or more weeks after discontinuation of the putative drug. Six deaths were not attributable to DI-LAD itself, as in our case. Treatment of DI-LAD usually consists of discontinuation of the offending medication. In this review, however, six patients were also treated with dapsone, corticosteroid or intravenous immunoglobulin. ${ }^{5}$

In the present report, chronology (onset of lesions 10 days after vancomycin introduction, and resolution 12 days after discontinuation) is compatible with the literature. In the cases associated with vancomycin reviewed by Fortuna et al., latency period ranged from 2 to 21 days and clinical remission occurred 1 to 21 days after drug discontinuation. ${ }^{3}$

The DIF pattern presented by our patient is classic in LAD, but $\mathrm{C} 3$ and IgM deposits can also be found in association with IgA. LAD histopathology is not specific and may be similar to $\mathrm{DH}$ and BP. A subepidermal blister with neutrophilic infiltrate is the most common description, but the infiltrate can be eosinophilic, mimicking $\mathrm{BP}^{4}$

Identification of the target antigens by immunoblotting has been performed in a few cases of DI-LAD. Different molecular weight proteins of the BMZ have been implicated (83-, 97-, 130-, 210, 230-, 285- (LAD285), 180Kda; NC16a domain of BP180; type VII collagen; $\alpha 3$ subunit of laminin-332). ${ }^{6,7}$ Because of the multiple possible antigens involved, indirect immunofluorescence on salt-split skin can be positive on the epidermal side, dermal side or both. ${ }^{4,8}$

DI-LAD pathogenesis has not been completely elucidated yet. Implicated drugs can cause disruption of self-tolerance by a hapten-mediated process or by structurally modifying proteins at the BMZ. Triggering events such as infections may act as co-factors. ${ }^{9}$
In 2012, Fortuna et al. reviewed the literature in journals indexed in PubMed and selected 52 cases of DI-LAD. Out of the total, $46.2 \%$ were related to vancomycin and $53.8 \%$ to other substances, especially captopril, trimethoprim/sulfamethoxazole, phenytoin and diclofenac. ${ }^{3}$ Chart 1 contains an updated list of drugs associated with LAD. ${ }^{1}$

Despite the considerable number of studies reporting association between LAD and medications, there is poor evidence

Chart 1: List of drugs associated with LAD in the literature up to 2015

\begin{tabular}{|ll}
\hline Vancomycin & \\
Captopril & Penicillin G \\
Trimethoprim/Sulfamethoxazole & Interferon / interleucina 2 \\
Phenytoin & Verapamil \\
Diclofenac & Vigabatrin \\
Amiodarone & Imipenem \\
Piroxicam & Ketoprofen \\
Naproxen & Carbamazepine \\
Acetaminophen & Amlodipine \\
Ceftriaxon & Candesartan/Eprosartan \\
Amoxicillin & Somatostatin \\
Atorvastatin & Buprenorphine \\
Lithium carbonate & Metronidazole \\
Gemcitabine & Moxifloxacin \\
Ampicilin & Sulfasalazine \\
Furosemide & Cefuroxime axetil \\
& Ampicillin/sulbactam \\
\end{tabular}

Adapted from: Chanal et al., 2013. ${ }^{1}$

Снавт 2: Naranjo algorithm for causality assessment between a drug and possible related adverse reactions

\begin{tabular}{|c|c|c|c|}
\hline Questions & YES & NO & UNKNOWN \\
\hline 1. Are there previous conclusive reports on this reaction? & +1 & 0 & 0 \\
\hline 2. Did the adverse event appear after the suspected drug was given? & +2 & -1 & 0 \\
\hline $\begin{array}{l}\text { 3. Did the adverse reaction improve when the drug was discontinued } \\
\text { or a specific antagonist was given? }\end{array}$ & +1 & 0 & 0 \\
\hline 4. Did the adverse reaction appear when the drug was readministered? & +2 & -1 & 0 \\
\hline 5. Are there alternative causes that could have caused the reaction? & -1 & +2 & 0 \\
\hline 6. Did the reaction reappear when a placebo was given? & -1 & +1 & 0 \\
\hline 7. Was the drug detected in any body fluid in toxic concentrations? & +1 & 0 & 0 \\
\hline $\begin{array}{l}\text { 8. Was the reaction more severe when the dose was increased or less } \\
\text { severe when the dose was decreased? }\end{array}$ & +1 & 0 & 0 \\
\hline $\begin{array}{l}\text { 9. Did the patient have a similar reaction to the same drug or similar } \\
\text { drugs in any previous exposure? }\end{array}$ & +1 & 0 & 0 \\
\hline 10. Was the adverse event confirmed by any objective evidence? & +1 & 0 & 0 \\
\hline
\end{tabular}

Adapted from: Naranjo et al., $1981 .^{10}$ 
of causality in the majority of them. Diagnosis usually relies on chronology and previously published cases. However, as these patients are often exposed to many concurrent medications, establishing a cause-effect relationship may be challenging. Challenge-dechallenge-rechallenge testing protocol should be the gold-standard procedure, but is not always feasible because of ethical and operational aspects. ${ }^{3} \mathrm{~A}$ reasonable option is the use of internationally accepted algorithms for causality assessment in adverse drug reactions (ADR), such as Naranjo algorithm (Chart 2). ${ }^{10}$
In the case we presented, vancomycin was considered to have induced LAD due to chronological plausibility, a Naranjo score of 4 (possible ADR) and previous conclusive reports on this reaction.

This report aims to draw attention to the possibility of LAD in cases of suspected TEN and to emphasize the need for a biopsy and DIF in all of them. The list of medications implicated in DI-LAD development has grown in recent years, but one has to be cautious in establishing causality. The use of algorithms to estimate the probability of ADR can aid in establishing a diagnosis.

\section{REFERENCES}

1. Chanal J, Ingen-Housz-Oro S, Ortonne N, Duong TA, Thomas M, Valeyrie-Allanore L, Lebrun-Vignes B, André C, Roujeau JC, Chosidow 0, Wolkenstein P. Linear IgA bullous dermatosis: comparison between the drug-induced and spontaneous forms. Br J Dermatol. 2013;169:1041-8.

2. Gabrielsen TO, Staerfelt $F$, Thune PO. Drug induced bullous dermatosis with linear IgA deposits along the basement membrane. Acta Derm Venereol. 1981;61:43941.

3. Fortuna G, Salas-Alanis JC, Guidetti E, Marinkovich MP. A critical reappraisal of the current data on drug-induced linear immunoglobulin $A$ bullous dermatosis: a real and separate nosological entity? J Am Acad Dermatol. 2012;66:988-94

4. Wojnarowska F, Venning VA. Immunobullous diseases. In: Burns T, Breathnach S, Cox N, Griffiths C, editors. Rook's: Textbook of Dermatology. 8th ed. Oxford: Blackwell Publishing; 2010. p. 40.45-40.51.

5. Kakar R, Paugh H, Jaworsky C. Linear IgA bullous disease presenting as toxic epidermal necrolysis: a case report and review of the literature. Dermatology. 2013;227:209-13.

6. Zenke $\mathrm{Y}, \mathrm{Nakano} \mathrm{T}$, Eto $\mathrm{H}$, Koga $\mathrm{H}$, Hashimoto $\mathrm{T}$. A case of vancomycin-associated linear IgA bullous dermatosis and IgA antibodies to the a3 subunit of laminin-332. Br J Dermatol. 2014;170:965-9.

7. Tashima S, Konishi K, Koga H, Hashimoto T. A case of vancomycin-induced linear IgA bullous dermatosis with circulating IgA antibodies to the NC16a domain of BP180. Int J Dermatol. 2014;53:e207-9.

8. Willsteed E, Bhogal B, Black M, McKee P, Wojnarowska F. Use of $1 \mathrm{M} \mathrm{NaCl}$ split skin in the indirect immunofluorescence of the linear IgA bullous dermatoses. $J$ Cutan Pathol. 1990;17:144-8.

9. Klein PA, Callen JP. Drug-induced linear IgA bullous dermatosis after vancomycin discontinuance in a patient with renal insufficiency. J Am Acad Dermatol. 2000;42:316-23

10. Naranjo CA, Busto U, Sellers EM, Sandor P, Ruiz I, Roberts EA, et al. A method for estimating the probability of adverse drug reactions. Clin Pharmacol Ther. 1981;30:239-45.

\author{
MAILING ADDRESS: \\ Amanda Regio Pereira \\ R. Borges Lagoa, 508 \\ Vila Clementino \\ 04038-001 - São Paulo - SP \\ Brazil \\ E-mail: amandaregiopereira@gmail.com
}

How to cite this article: Pereira AR, de Moura LHB, Pinheiro JRS, Pasin VP, Enokihara MMSS, Porro AM. Vancomycinassociated linear IgA disease mimicking toxic epidermal necrolysis. An Bras Dermatol. 2016;91(5 Supl 1):S35-8. 